\title{
Effects of Standardized Home Training in Patients with Cognitive Impairment following Geriatric Rehabilitation: A Randomized Controlled Pilot Study
}

\author{
Klaus Hauer $^{\mathrm{a}}$ Phoebe Ullrich $^{\mathrm{a}} \quad$ Ilona Dutzi $^{\mathrm{a}}$ Rainer Beurskens ${ }^{\mathrm{a}}$ Sylvia Kern ${ }^{\mathrm{b}}$ \\ Jürgen Bauer ${ }^{\mathrm{a}}$ Michael Schwenk ${ }^{\mathrm{a}}$ \\ aDepartment of Geriatric Research, AGAPLESION Bethanien Hospital, University of Heidelberg, Heidelberg, and \\ ${ }^{b}$ Alzheimer Gesellschaft Baden Württemberg, Stuttgart, Germany
}

\section{Keywords}

Dementia - Cognitive impairment · Home-based exercise . Geriatric rehabilitation - Balance $\cdot$ Strength $\cdot$ Physical activity

\begin{abstract}
Background: Post-ward geriatric rehabilitation programs have hardly been developed and validated, which leaves a substantial gap in rehabilitative care in older adults and hinders full exploitation of maintained, but often unrecognized rehabilitation potentials. Geriatric rehabilitation patients with cognitive impairment represent a highly vulnerable population which is often affected by a lack of an ongoing support at the intersection between ward-based and postward rehabilitation. Objective: To determine the effect of a standardized home-based training program in geriatric patients with cognitive impairment following ward-based rehabilitation. Methods: A randomized controlled, singleblinded intervention trial (RCT) with wait list control design was used. Geriatric patients ( $n=34$; age: $81.9 \pm 5.7$ years) with cognitive impairment (MMSE: $18.8 \pm 4.7$ ), identified by predefined in- and exclusion criteria, were consecutively recruited from a geriatric rehab ward. Patients in the interven-
\end{abstract}

\section{KARGER}

(C) 2017 S. Karger AG, Basel

E-Mail karger@karger.com

www.karger.com/ger tion group (IG, $n=17$ ) performed a 6-week strength and functional home training. The control group (CG, $n=17$ ) started an identical training 6 weeks later with an initial usual care period during the intervention for the IG. Functional performance (Short Physical Performance Battery; SPPB), clinically relevant functional deficits (Performance Oriented Assessment; POMA), and physical activity (Assessment of Physical Activity For Older Persons questionnaire; APAFOP) represented primary outcome measurements complemented by additional secondary outcome parameters. Results: The IG significantly increased functional performances in SPPB (total score: $p=0.012$; chair rise: $p=0.007$, balance: $p=$ 0.066 ), reduced gait and balance deficits in POMA (total score: $p=0.006$; balance: $p=0.034$; gait: $p=0.019$ ), and increased physical activity (APAFOP; $p=0.05$ ) compared to the $C G$. Effect sizes showed medium to large effects for significant parameters (eta ${ }^{2}=0.14-0.45$ ). Training benefits and adherence were more pronounced following the immediate onset of post-ward training compared to a delayed start (eta $\left.{ }^{2}=0.06-0.23\right)$. Conclusion: Results of this pilot study show that a feasible and easy to handle, home-based reha-

Klaus Hauer and Phoebe Ullrich share first authorship.
Prof. Dr. Klaus Hauer

Department of Geriatrics, AGAPLESION Bethanien Hospital

University of Heidelberg, Rohrbacher Strasse 149

DE-69126 Heidelberg (Germany)

E-Mail khauer@bethanien-heidelberg.de 
bilitation program increased functional performance and physical activity in a vulnerable, multimorbid patient group with cognitive impairment, in particular when the post-ward training onset was not postponed.

(c) 2017 S. Karger AG, Basel

\section{Introduction}

Although cognitive impairment (CI) is prevalent in $30-80 \%$ of patients admitted to inpatient rehabilitation, only a limited number of rehabilitation programs have been developed and evaluated for this vulnerable patient group. This is surprising since this group shows an increased number of risk factors affecting their health status compared to cognitively intact patients. Those are: higher multimorbidity [1], loss of independence [2], and increased need of care and institutionalization [3]. CI is associated with an increased risk for a negative rehabilitation outcome, a smaller functional recovery during inpatient rehabilitation and a lower functional status at discharge [4]. Further, if transitional care interventions following inpatient rehabilitation are provided, persons with the highest risk (e.g., with CI, comorbidities) are often excluded [5].

Besides their insufficiently exploited rehabilitation potential, previous research indicated that rehabilitation programs are generally suitable to improve functional performance in cognitively impaired persons (cf. [6] for a review). Therefore, effective rehabilitation programs at the transition from geriatric inpatient rehabilitation to the home environments are needed for these patients [5]. Effective post-ward rehabilitation programs may not only increase the ability to perform activities of daily living (ADL; as the most important predictor of societal costs of care of community-dwelling patients) and improve functional performances and mobility, but moreover they may allow proper preservation of autonomy (a most appraised goal of geriatric rehabilitation). Particularly, patients with CI may have limited access to medical services including post-ward rehabilitation (e.g., transport to participate in rehabilitation programs is considered a typical barrier) [7].

Previous findings indicate that home-based programs are feasible in community-dwelling older individuals, which can be considered high-functioning cohorts of older adults compared to geriatric patients with CI [8-13], showing adherence rates of $72-91 \%$ in the training groups. However, beneficial effects of the presented programs are inconsistent with some studies indicating per- formance improvements [8], while others showed decelerated deterioration of physical function and mobility [9] or did not find any training-related effects [11-13]. Further, the respective studies showed methodological inconsistencies, e.g. using questionnaire-based assessment methods to register physical activity and mobility [1012], did not provide sufficient information on motor performance measures [10] or did not register key motor features (i.e., gait, balance, strength) as primary endpoints $[10,11]$.

Publications on home-based exercise programs focusing on patients with CI following discharge from a geriatric hospital are scarce. This is surprising since our research group as well as others were able to show that supervised ward-based [14] and post-ward programs [15] are effective in improving strength, motor function, and physical activity in this specific population. However, solutions to increase sustainability of rehabilitation programs will have to be low-cost as rapidly increasing budgets for medical care in older persons already endanger adequate and effective health care. To date, interventions at the transition from geriatric inpatient rehabilitation to the home environments have been evaluated in heterogeneous patient populations [16-20]. Most programs included cognitively intact patients $[16,17]$, while only few implemented subgroups of cognitively impaired patients [18-20] and found conflicting results. Some studies reported beneficial effects of home-based training on measures of motor performance $[16,18,20]$, while others did not [17]. Further, behavioral aspects such as physical activity, which is crucial for the sustainability of rehabilitation programs and a prominent indicator of durable behavioral change, have hardly been investigated in recent studies. Only 2 studies registered physical activity levels using questionnaires in community-dwelling older adults $[8,13]$, but none found intervention-related improvements in physical activity levels of older adults living at home. Another not yet thoroughly examined topic is the timing of post-ward rehabilitation in patients with CI. Timely outpatient follow-up is a key component of transitional care models that have been successful in reducing readmission rates [21]. Due to legal reconciliation, reconstruction works at the patients' homes to establish a disability-friendly environment or specific transitional measures (i.e., short-term care), the transition from the hospital back into their home environments is often delayed, and rehabilitative actions are postponed. However, research on rehabilitation onset in the group of geriatric patients with CI is lacking, and it remains unknown whether a timely onset of post-ward rehabilitation im-
496

Gerontology 2017;63:495-506

DOI: $10.1159 / 000478263$
Hauer/Ullrich/Dutzi/Beurskens/Kern/ Bauer/Schwenk 
proves functional outcomes in this vulnerable group. In elderly patients after cardiac surgery, early rehabilitation during inpatient rehabilitation increased gait autonomy at the end of the rehabilitation program (more than $90 \%$ of patients submitted to early rehabilitation showed significant increases in walking distance) [22]. Further, early cardiac rehabilitation (i.e., within 28 days of admission) significantly improved functional status (i.e., gait capacity and fitness level) compared to a delayed rehabilitation start (i.e., 29-365 days following admission) in patients with acute coronary syndrome [23]. That is, for every 1 -day delay, patients were $1 \%$ less likely to improve their motor performance. Similarly, a systematic review by Haykowski et al. [24] yielded evidence that each week that cardiac rehabilitation is delayed required an additional month of training to achieve the same level of benefit.

Therefore, the aim of this study was to develop and validate an easy-to-implement and low-cost post-ward rehabilitation program, which is feasible for frail, multimorbid patients suffering from CI at the transition from geriatric inpatient rehabilitation to their home environments. We hypothesized that the training program is (a) suitable and feasible to be implemented at patients' homes, (b) improves key motor performance (i.e., gait performance, balance performance, and strength), (c) increases physical activity within the observation period, and (d) an early onset of training is better suited to improve training adherence and effectiveness following hospital discharge compared to a later onset of training.

\section{Methods}

\section{Study Design}

We present a randomized, controlled pilot study with wait list control group design. Patients in the intervention group (IG) took part in a standardized 6-week home training. Patients assigned to the control group (CG) received usual care and were not contacted during the first observational period (period: T1-T2). After the first training period, CG started to exercise according to the wait list control design (period: T2-T3) to document the effect of delayed onset of training. The study conforms to the Helsinki Declaration and was approved by the ethic committee of the Medical Department of the University of Heidelberg.

\section{Participants}

Patients admitted to rehabilitation at a geriatric hospital (AGAPLESION Bethanien Hospital, Heidelberg, Germany) between 10/2011 and 7/2012 were consecutively screened for predefined inclusion criteria: (1) Mini-Mental State Examination (MMSE) score $<24$, (2) age $>65$ years, (3) ability to stand or walk $5 \mathrm{~m}$ without support, (4) no severe somatic or psychiatric disease, (5) no premature termination of rehabilitation period, (6) resi- dence $<35$ kilometers to the study center, (7) no simultaneous participation in other studies, (8) written informed consent. A person not involved in study organization randomly assigned patients to the study groups.

\section{Intervention}

The home training program was specifically developed for the target sample to be feasible and effective in multimorbid, frail older adults. The main contents of the training program were postural control and strength, each addressed by 3 exercises. The training intervention was derived from an exercise program developed by our research group, which was feasible and effective to increase strength and functional performances in patients with CI [15] and has additionally been used on a website providing interactive motor training and functional assessment for persons with CI (http://bewegung-bei-demenz.de/). We did not include specific gait training as we felt that an unsupported gait task might represent a high fall risk as compared to supported balance and transfer tasks.

During the first training session, the program was explained and supervised by a professional trainer experienced in communication strategies for cognitively impaired persons. Dementiaspecific strategies included emotional aspects such as reassurance of training progress, positive feedback, and personal care/empathy, as described in patient-centered communication techniques developed for individuals with dementia [25]. Further, simple structures of motor tasks and haptic support during motor actions were provided, and the use of verbal cues instead of complex verbal instructions was fostered by the trainer [26]. To foster participation and to provide assistance, exercises were illustrated on a large poster $(60 \times 120 \mathrm{~cm}$; Fig. 1) at patients' homes, and a comprehensive manual of key elements for each exercise (including safety instructions, e.g., using a chair or a nearby wall for support during balance tasks) was developed and provided to the participants. Postural balance tasks included standing in progressively challenging positions (side by side stance, semi-tandem stance, tandem stance). Strength exercises targeted basic ADLrelated key motor functions, including functional strength (such as ankle lifts, chair rises, and stair rises). Participants were instructed to perform 2 series of 7-10 repetitions for each exercise. Balance positions were maintained for $30 \mathrm{~s}$. Progression of training was facilitated by increasing task difficulty (e.g., narrowing standing positions, reduction of postural stabilization by reducing handgrip) and by increasing the workload (number of repetitions in strength-related tasks, single vs. double stand, reducing arm support). Participants were instructed to exercise at least once per day, but encouraged to exercise as often as possible. Training intensity was adjusted to individuals' performance levels, and participants were instructed to stop exercising or reduce difficulty when feeling exhausted or overtaxed. Subjects were contacted weekly to document training adherence, to increase compliance and to adjust individual training levels/tasks based on a manual delivered discussed at beginning of intervention. Proxies or carers were informed about the program and instructed to support participants. No other care or therapy except for the intervention program was offered to the participants in both groups. During the first observation period (T1-T2), CG did not receive any additional support or contact except for baseline and postinterventional assessment. 
AGAPLESION

BETHANIEN KRANKENHAUS

urasemeco

\section{$2 \times 3=$ That's the Key}

Balance

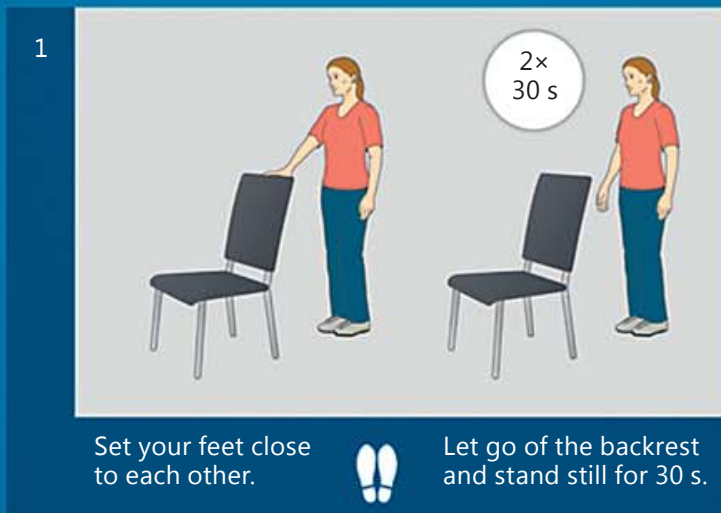

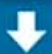

2

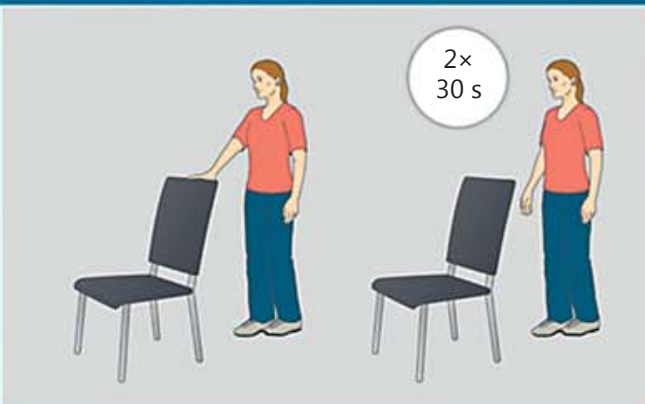

Set the tip of one of your feet at the side of the other.

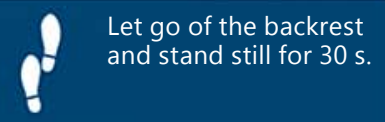

$\checkmark$

3

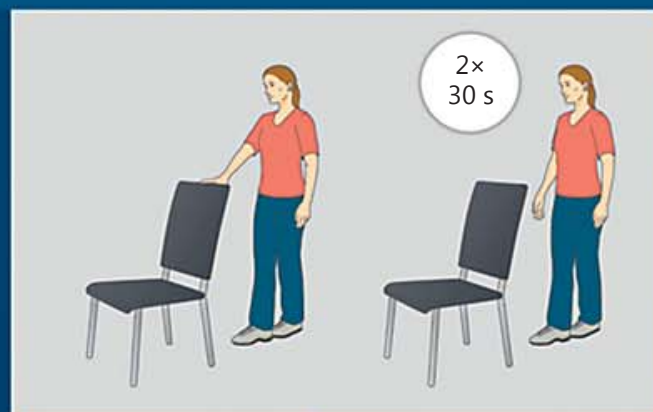

Set one of your feet straight ahead of the other one.

Let go of the backrest and stand still for $30 \mathrm{~s}$.

1

Strength

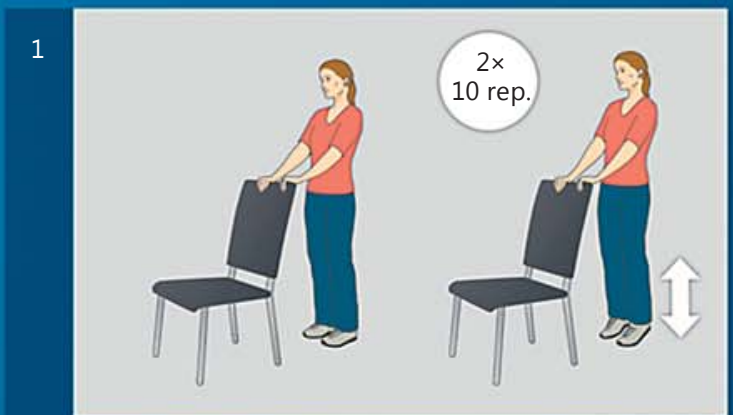

Set your feet next to each other and keep hold of the backrest.

Of back 10 times.

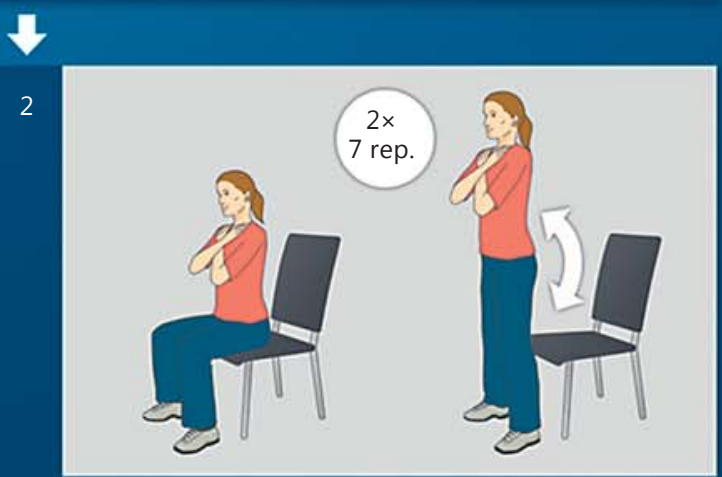

Sit down on a chair with your arms crossed.
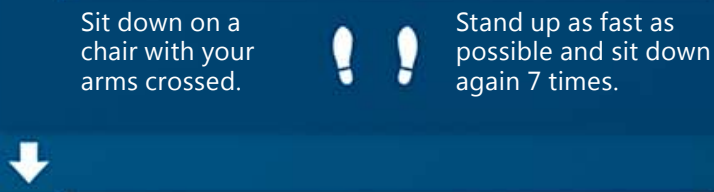

3

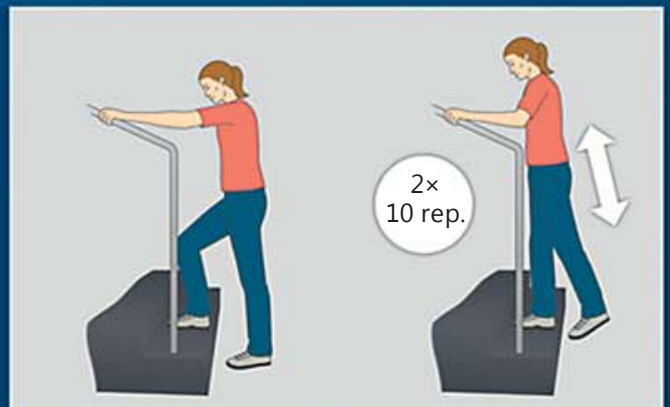

Set one of your feet on the first step and the other in front of the step.
Mount the step and lower yourself 10 times. f 


\section{Measurements}

Standardized measurements were performed before randomization at the end of ward-based rehabilitation (T1), at the end of the 6-week training period (T2, RCT design), and after a 6-week follow-up period (T3; training for the initial CG). Assessors were trained in documenting motor performances in patients with $\mathrm{CI}$ [15] and were blinded to randomization.

Clinical characteristics, i.e. functional status (Barthel index), social status (living independently/with spouse, support by proxies/public care service, proxies living in same facility, or 24-h care service), psychological status (Geriatric Depression Scale, GDS) [27], and cognitive status (MMSE) [28] were documented at baseline. Functional performances were assessed by well-established, validated tests: the performance-based Short Physical Performance Battery (SPPB), including subtests of chair rise, balance and gait performance [29] and Tinetti's Performance Oriented Motor Assessment (POMA) [30], including subtest on gait and balance. In addition, results of the SPPB gait test and chair rise test were documented as absolute duration (seconds) not transcribed into to SPPB scoring to assess qualitative performance.

Physical activity was documented by the Assessment of Physical activity for Older Persons (APAFOP), a questionnaire developed and validated for persons with CI [31]. APAFOP captured total physical activity by multiplication of metabolic equivalent levels of activities and times spend in the respective activity over a period of $24 \mathrm{~h}$ using specific memory techniques to increase the accuracy of reports. Adherence to the training intervention was documented as number of training sessions within the training period, and training-related adverse events were prospectively documented by standardized weekly telephone calls [32].

Technical measures focused on Sit-to-Stand (STS) performances and were assessed using accelerometer-based body fixed sensors (DynaPort ${ }^{\circledR}$ Hybrid, McRoberts, The Hague, the Netherlands) which have been validated in geriatric patients [33]. For STS, patients performed the chair rise test representing an established functional assessment in older adults [29]. We analyzed parameters with a high impact on successful STS performance; that is total duration and duration of hip extension/flexion as well as maximum angular velocity (during hip extension/flexion) of both the stand-to-sit as well as sit-to-stand movements. Because of technical reasons (i.e., availability of the assessment unit) as well as floor effects within the study group, only a subsample of patients was included in the accelerometer-based assessment.

\section{Statistical Analysis}

An a priori power analysis using a repeated measure analysis of variance (ANOVA) design including 2 groups and 2 repeated measurements yielded a total sample size of $n=28$ ( $\alpha=0.05$; critical $F=4.23$; power $=0.8$ ). The effect size is based on an RCT comparing 12 weeks of group-based resistance/functional training and low-intensity placebo activity in 122 older adults with CI [17]. Results showed an average improvement of $26 \%$ in functional performance (POMA score; effect size: eta $^{2}=0.22$ ). Estimating a dropout of $\sim 20 \%$, the sample size was estimated to be 34 participants.

Fig. 1. Exercise poster for exercises used in the study. Displayed are balance exercises (i.e., side by side, semi-tandem, and tandem stance) on the right and strength exercises (i.e., calf raise, sit to stand transfer, and stair rise) on the left side. rep., repeats.

Home Training in Cognitively Impaired

Patients
Table 1. Baseline comparison of study groups

\begin{tabular}{lccl}
\hline Parameter & IG $(n=17)$ & CG $(n=17)$ & $p$ value \\
\hline Age, years & $81.4 \pm 6.6$ & $83.3 \pm 5.7$ & 0.379 \\
Weight, kg & $67.0 \pm 13.7$ & $68.5 \pm 15.7$ & 0.772 \\
Gender, female & $65 \%$ & $71 \%$ & - \\
Ward rehabilitation, days & $22.9 \pm 6.5$ & $21.5 \pm 8.9$ & 0.615 \\
MMSE score & $19.5 \pm 4.6$ & $18.2 \pm 4.4$ & 0.401 \\
GDS score & $4.0 \pm 3.4$ & $5.6 \pm 2.3$ & 0.390 \\
ADL-Barthel score & $71.2 \pm 17.7$ & $67.1 \pm 17.1$ & 0.496 \\
POMA score & $16.4 \pm 5.8$ & $17.7 \pm 6.2$ & 0.464 \\
SPPB score & $4.5 \pm 2.7$ & $4.5 \pm 2.8$ & 0.999 \\
APAFOP score & $26.0 \pm 1.2$ & $25.7 \pm 1.1$ & 0.342
\end{tabular}

$p$ values represent comparison between study groups for mean \pm SD at baseline. MMSE, Mini-Mental State Examination; GDS, Geriatric Depression Scale; ADL, activities of daily living; SPPB, Short Physical Performance Battery; POMA, Performance Oriented Motor Assessment; APAFOP, Assessment of Physical Activity in Older Persons.

Exploratory data analysis determined the variability and distribution of outcome variables. Unpaired $t$ test and $\chi^{2}$ test were used for baseline comparison and comparisons of training/contact frequency. Primary study endpoints were the total scores of SPPB, POMA, and APAFOP. Secondary endpoints were subscores of the SPPB (chair rise, balance, gait) including duration of walking and chair rise during subtests of SPPB, and results of technical STS assessment including movement durations (total, flexion, extension), and maximum angular velocities (flexion/extension). Between-group differences were analyzed by ANOVAs with repeated measures for intervention effects (T1-T2) within the RCT design. To examine the effects of an early versus delayed training onset, ANOVAs with repeated measures for intervention effects (IG: T1T2; CG: T2-T3) within the wait list design were used. Time $\times$ Group effects were given for between-group analysis of intervention effects. Time effects were given to document time-related effects only. Effect sizes were documented as partial eta ${ }^{2}$, a measure sensitive to various kinds of linear and nonlinear relationships. Values ranging from 0.01 to 0.06 indicate low; values from 0.06 to 0.25 medium, and values above 0.25 large effect sizes [34]. All analyses were calculated using Statistical Package for Social Sciences (SPSS) version 23.0 (IBM Corp., New York, NY, USA), and significance levels were set at $\alpha=5 \%$.

\section{Results}

Among 561 patients screened for participation, 34 (age range: 69-94 years) matching our predefined inclusion criteria were included in the study. Primary causes for exclusion were cognitive status $(n=292)$, readmission to the hospital $(n=57)$, and institutionalization $(n=56)$. Six

Gerontology 2017;63:495-506 499 


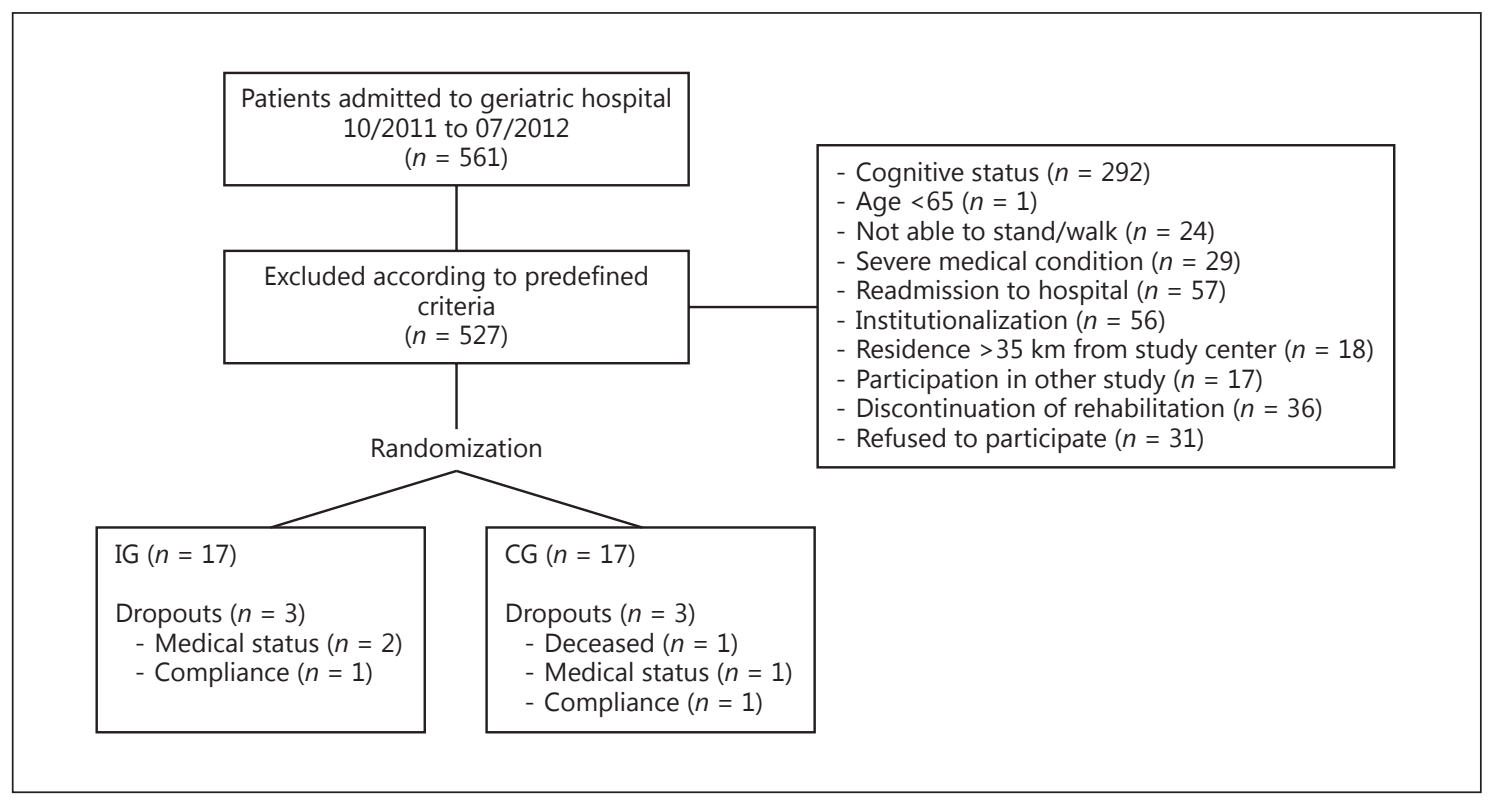

Fig. 2. Flowchart of patient recruitment. A total of 561 patients were screened for inclusion; 527 were excluded according to predefined exclusion criteria; 34 were randomized into the study groups (intervention group [IG]: $n=17$, control group [CG]: $n=17)$.

patients dropped out after randomization within the first training period (IG: $n=3$; CG: $n=3$ ). Figure 2 shows the recruitment procedure according to the CONSORT statement [35]. IGs did not differ at baseline, indicating a successful randomization (Table 1). Compliance to training was high and no adverse training-related events occurred.

\section{Effects of Home-Based Training}

The training intervention significantly increased performance in primary study endpoints for motor performance as well as for physical activity (Table 2). Results of the SPPB indicated overall, significant improvement in key motor performances (increase in SPPB total score by $40 \%)$. In subtests specifically addressed in the training program (i.e., chair rise and balance), performance significantly increased in IG compared to CG. In the subtest not specifically addressed during training (i.e., walking), performance improved in both groups with a clinically relevant increase in the IG resulting in significant Time but not Time $\times$ Group effects. SPPB results expressed as absolute time-based performance and not scoring results further confirmed results based on scoring. Similarly, results of the POMA indicated a significant overall reduction of gait and balance deficits (increase in total POMA score by $24 \%$ ) as well as a reduction in both subperfor- mances of gait and balance in IG compared to CG. Effect sizes ranging from 0.23 to 0.45 for the significant results of the SPPB and from 0.16 to 0.25 for the POMA indicated medium to large effects of the intervention (Table 2), documenting not only statistically significant but clinically relevant improvements [36]. When training results were combined for both training groups (immediate training in IG and delayed training in CG) to increase sample size and statistical power, the above-documented results were confirmed and ANOVAs additionally indicated significant performance improvements in subscores of SPPB $\left(+18 \%, p=0.001\right.$, eta ${ }^{2}=0.33$ for SPPB balance; $+23 \%, p=0.043$, eta ${ }^{2}=0.15$ for SPPB - gait).

\section{Sensor-Based Outcome Measures}

For a subsample of participants, additional sensorbased assessments were performed during STS (Table 3). Despite the very small number of participants, interventional effects led to significant improvements during stand-to-sit transfers (total duration, duration of extension/flexion, and maximal angular velocity during extension: $p=0.006-0.044 ;$ eta $\left.^{2}=0.35-0.55\right)$ and during sit-tostand transfers (extension duration: $p=0.018$, eta $^{2}=0.44$ ) based on very large effect sizes achieved. 
Table 2. Effects of intervention

\begin{tabular}{|c|c|c|c|c|c|c|c|c|}
\hline & \multicolumn{2}{|c|}{$\mathrm{IG}(n=14)$} & \multicolumn{2}{|c|}{$\mathrm{CG}(n=14)$} & \multicolumn{2}{|c|}{ Time effects } & \multicolumn{2}{|c|}{ Time $\times$ Group effects } \\
\hline & $\mathrm{T} 1$ & $\mathrm{~T} 2$ & $\mathrm{~T} 1$ & $\mathrm{~T} 2$ & $p$ value & ES & $p$ value & ES \\
\hline \multicolumn{9}{|l|}{ Primary outcomes } \\
\hline SPPB - total, score & $5.0 \pm 2.7$ & $7.0 \pm 2.7$ & $4.4 \pm 2.9$ & $4.7 \pm 2.4$ & 0.001 & 0.35 & 0.010 & 0.23 \\
\hline POMA - total, score & $18.6 \pm 7.5$ & $23.0 \pm 4.4$ & $18.4 \pm 7.0$ & $18.6 \pm 5.2$ & 0.003 & 0.29 & 0.006 & 0.25 \\
\hline APAFOP, score & $26.2 \pm 1.1$ & $27.2 \pm 2.0$ & $25.7 \pm 1.2$ & $25.7 \pm 1.2$ & 0.037 & 0.16 & 0.050 & 0.14 \\
\hline \multicolumn{9}{|l|}{ Secondary outcomes } \\
\hline SPPB - chair rise, score & $0.6 \pm 0.8$ & $1.5 \pm 1.4$ & $0.7 \pm 1.3$ & $0.7 \pm 1.1$ & 0.007 & 0.25 & 0.007 & 0.25 \\
\hline SPPB - balance, score & $2.9 \pm 0.9$ & $3.6 \pm 0.7$ & $2.6 \pm 0.7$ & $2.7 \pm 0.7$ & 0.024 & 0.18 & 0.066 & 0.12 \\
\hline SPPB - gait, score & $1.4 \pm 1.3$ & $1.9 \pm 1.3$ & $1.1 \pm 1.4$ & $1.3 \pm 1.1$ & 0.040 & 0.15 & 0.395 & 0.03 \\
\hline $\mathrm{SPPB}$ - gait speed, $\mathrm{m} / \mathrm{s}^{\mathrm{a}}$ & $0.5 \pm 0.2$ & $0.6 \pm 0.2$ & $0.4 \pm 0.2$ & $0.4 \pm 0.2$ & 0.069 & 0.19 & 0.153 & 0.12 \\
\hline SPPB - chair rise, $\mathrm{s}^{\mathrm{b}}$ & $20.9 \pm 5.7$ & $16.0 \pm 4.0$ & $19.1 \pm 9.8$ & $24.4 \pm 12.0$ & 0.912 & $<0.01$ & 0.009 & 0.45 \\
\hline POMA - balance, scores & $11.0 \pm 3.4$ & $13.3 \pm 1.7$ & $11.5 \pm 3.4$ & $11.7 \pm 1.8$ & 0.012 & 0.22 & 0.034 & 0.16 \\
\hline POMA - gait, scores & $7.6 \pm 4.5$ & $9.7 \pm 2.8$ & $6.9 \pm 3.9$ & $6.9 \pm 3.9$ & 0.019 & 0.19 & 0.019 & 0.19 \\
\hline
\end{tabular}

Presented is a between-group comparison for effects of intervention for "Time $\times$ Group" and "Time" effects. $p$ values are given for ANOVAs with repeated measures, effect sizes are given as partial eta ${ }^{2}$. IG, intervention group; CG, control group; POMA, Performance Oriented Motor Assessment; SPPB, Short Physical Performance Battery; APAFOP, Assessment of Physical Activity in Older Persons; ES, effect size. Figures in bold indicate significant $p$ values and ES with moderate to large effect $(>0.06) .{ }^{a}$ IG $(n=9)$, CG $(n=9)$. ${ }^{b}$ IG $(n=9)$, CG $(n=5)$.

Table 3. Effects of intervention (sensor-based measures)

\begin{tabular}{|c|c|c|c|c|c|c|c|c|}
\hline & \multicolumn{2}{|l|}{ IG $(n=8)$} & \multicolumn{2}{|l|}{$\mathrm{CG}(n=5)$} & \multicolumn{2}{|c|}{ Time effects } & \multicolumn{2}{|c|}{ Time $\times$ Group effects } \\
\hline & $\mathrm{T} 1$ & $\mathrm{~T} 2$ & $\mathrm{~T} 1$ & $\mathrm{~T} 2$ & $p$ value & ES & $p$ value & ES \\
\hline \multicolumn{9}{|l|}{ Sit-to-stand } \\
\hline Duration, s & $1.8 \pm 0.4$ & $1.4 \pm 0.2$ & $2.0 \pm 0.6$ & $2.5 \pm 0.9$ & 0.706 & 0.02 & 0.064 & 0.30 \\
\hline Flex. duration, s & $0.9 \pm 0.2$ & $0.8 \pm 0.2$ & $1.0 \pm 0.4$ & $1.1 \pm 0.4$ & 0.745 & 0.02 & 0.451 & 0.06 \\
\hline Ext. duration, s & $0.9 \pm 0.3$ & $0.6 \pm 0.1$ & $0.9 \pm 0.2$ & $1.4 \pm 0.6$ & 0.334 & 0.13 & 0.018 & 0.44 \\
\hline Max. ang. vel. flex., $\%$ s & $86.2 \pm 35.9$ & $106.0 \pm 54.5$ & $74.0 \pm 21.1$ & $60.4 \pm 10.0$ & 0.893 & $<0.01$ & 0.239 & 0.14 \\
\hline Max. ang. vel. ext., $\%$ s & $42.2 \pm 22.7$ & $37.7 \pm 17.5$ & $46.9 \pm 26.5$ & $41.4 \pm 15.4$ & 0.364 & 0.12 & 0.919 & 0.001 \\
\hline \multicolumn{9}{|l|}{ Stand-to-sit } \\
\hline Duration, s & $2.2 \pm 0.5$ & $1.5 \pm 0.3$ & $2.0 \pm 0.8$ & $2.4 \pm 0.7$ & 0.990 & 0.00 & 0.014 & 0.47 \\
\hline Flex. duration, s & $1.1 \pm 0.3$ & $0.7 \pm 0.2$ & $1.0 \pm 0.4$ & $1.3 \pm 0.3$ & 0.887 & $<0.01$ & 0.015 & 0.46 \\
\hline Ext. duration, s & $1.0 \pm 0.2$ & $0.8 \pm 0.2$ & $0.9 \pm 0.4$ & $1.1 \pm 0.4$ & 0.837 & 0.01 & 0.044 & 0.35 \\
\hline Max. ang. vel. flex., $\%$ s & $41.1 \pm 13.8$ & $46.5 \pm 27.1$ & $36.8 \pm 17.7$ & $31.5 \pm 12.4$ & 0.998 & 0.00 & 0.369 & 0.08 \\
\hline Max. ang. vel. ext., \%/s & $75.3 \pm 22.6$ & $107.7 \pm 50.1$ & $81.8 \pm 39.0$ & $52.5 \pm 10.3$ & 0.677 & 0.03 & 0.006 & 0.55 \\
\hline
\end{tabular}

Presented are results of STS performance during the chair stand test for ANOVAs with repeated measures for between-group comparison (Time $\times$ Group effect) according to the RCT design and Time effects for motor effects of intervention. Effect size is given as partial eta ${ }^{2}$. IG, intervention group; CG, control group; flex., hip flexion phase; ext., hip extension phase; max. ang. vel., maximum angular velocity; ES, effect sizes. Figures in bold indicate significant $p$ values and ES with moderate to large effect $(>0.06)$.

Home Training in Cognitively Impaired Patients
Gerontology 2017;63:495-506 DOI: $10.1159 / 000478263$ 
Table 4. Immediate versus delayed onset of training

\begin{tabular}{|c|c|c|c|c|c|c|c|c|}
\hline & \multicolumn{2}{|c|}{ Immediate $(n=14)$} & \multicolumn{2}{|c|}{ Delayed $(n=13)$} & \multicolumn{2}{|c|}{ Time effects } & \multicolumn{2}{|c|}{ Time $\times$ Group effects } \\
\hline & $\mathrm{T} 1$ & $\mathrm{~T} 2$ & $\mathrm{~T} 2$ & T3 & $p$ value & ES & $p$ value & ES \\
\hline \multicolumn{9}{|l|}{ Primary outcomes } \\
\hline SPPB total, score & $5.0 \pm 2.7$ & $7.0 \pm 2.7$ & $4.4 \pm 2.1$ & $5.2 \pm 2.3$ & $<0.001$ & 0.47 & 0.068 & 0.13 \\
\hline POMA total, score & $18.6 \pm 7.5$ & $23.0 \pm 4.4$ & $18.2 \pm 5.1$ & $18.6 \pm 4.9$ & 0.004 & 0.29 & 0.016 & 0.21 \\
\hline APAFOP, score & $26.2 \pm 1.1$ & $27.2 \pm 2.0$ & $25.6 \pm 1.2$ & $26.0 \pm 1.3$ & 0.011 & 0.23 & 0.258 & 0.05 \\
\hline \multicolumn{9}{|l|}{ Secondary outcomes } \\
\hline SPPB - chair rise, score & $0.6 \pm 0.7$ & $1.5 \pm 1.3$ & $0.7 \pm 1.3$ & $0.9 \pm 1.3$ & 0.002 & 0.33 & 0.053 & 0.14 \\
\hline SPPB - balance, score & $2.9 \pm 0.9$ & $3.6 \pm 0.6$ & $2.6 \pm 0.7$ & $3.0 \pm 0.7$ & 0.002 & 0.33 & 0.384 & 0.03 \\
\hline SPPB - gait, score & $1.4 \pm 1.3$ & $1.9 \pm 1.3$ & $1.1 \pm 0.9$ & $1.3 \pm 0.9$ & 0.043 & 0.15 & 0.440 & 0.02 \\
\hline $\mathrm{SPPB}$ - gait speed, $\mathrm{m} / \mathrm{s}^{\mathrm{a}}$ & $0.5 \pm 0.2$ & $0.6 \pm 0.2$ & $0.3 \pm 0.2$ & $0.4 \pm 0.2$ & 0.037 & 0.23 & 0.311 & 0.06 \\
\hline SPPB - chair rise, $\mathrm{s}^{\mathrm{b}}$ & $20.9 \pm 5.7$ & $16.0 \pm 4.0$ & $25.1 \pm 12.1$ & $17.4 \pm 6.5$ & 0.001 & 0.60 & 0.351 & 0.07 \\
\hline POMA - balance, score & $11.0 \pm 3.4$ & $13.3 \pm 1.7$ & $11.6 \pm 1.9$ & $11.8 \pm 2.0$ & 0.004 & 0.28 & 0.011 & 0.23 \\
\hline POMA - gait, score & $7.6 \pm 4.5$ & $9.7 \pm 2.8$ & $6.5 \pm 3.7$ & $6.8 \pm 3.6$ & 0.023 & 0.19 & 0.082 & 0.12 \\
\hline
\end{tabular}

Presented is a between-group comparison for effects of training onset for "Time $\times$ Group" and time-related effects. $p$ values are given for analysis of variance, repeated measures, effect sizes are given as partial eta ${ }^{2}$. POMA, Performance Oriented Motor Assessment; SPPB, Short Physical Performance Battery; APAFOP, Assessment of Physical Activity in Older Persons; ES, effect sizes. Figures in bold indicate significant $p$ values and ES with moderate to large effect $(>0.06)$. ${ }^{\text {a }}$ Immediate $(n=9)$, delayed $(n=10)$. ${ }^{\mathrm{b}}$ Immediate $(n=9)$, delayed $(n=6)$.

\section{Immediate versus Delayed Onset of Training}

Adherence rates significantly differed between an immediate and delayed onset of training $\left(p=0.025\right.$, eta ${ }^{2}=$ $0.19)$. Patients in the immediate group completed $40.9 \pm$ 25.9 training sessions (i.e., average of 0.97 training sessions per day) while patients in the delayed group conducted $20.8 \pm 16.5$ training sessions on average (i.e., 0.49 training sessions per day on average). Regarding functional performance, significant improvements or at least a trend to improved functional performance in the immediate training group compared to the delayed training group could be observed ( $p=0.011-0.082$; eta $^{2}=0.12-$ 0.23 ; Table 4 ). This finding is supported by medium effect sizes found in 7 out of 11 parameters $\left(\right.$ eta $^{2}=0.06-0.23$ ) that were present despite the rather small sample size. Further, each registered parameter showed a significant effect of Time $\left(p=0.001-0.043\right.$, eta $\left.{ }^{2}=0.15-0.60\right)$, indicating that both training groups increased motor performance and physical activity following the home-based training.

\section{Discussion}

This study investigated the effects of a home-based post-ward training program on functional performance and physical activity in patients with CI. Our main results can be summarized as follows: (1) the program was feasible to be implemented in cognitively-impaired patients following hospital discharge, (2) the training intervention significantly improved key motor features and increased physical activity levels, and (3) training-related performance improvements benefit from an early onset of training after hospital discharge.

\section{Feasibility and Safety of the Home-Based Training}

Our results are in line with previous studies including home-based exercise in patients with Alzheimer disease [8] and with studies investigating supervised ward-based and post-ward [15] interventions in patients with CI. Findings indicate that a home-based training program is feasible and well-accepted in this vulnerable target population. Patients in our study conducted 0.97 training sessions per day on average and thus met the prospected training goal. Even those who trained less during the training period reached at least $60 \%$ of the intended training sessions while others conducted up to $300 \%$ (range: 26-129 session per day). In the present study, a motivational approach (i.e., assessors and supervisors used a dementia-specific communication approach) was used [15], which is based on established care models such as validation, personal assistance by home visits and weekly phone calls, easy to understand, visualized training instructions on a poster, individually tailored exercises al-
502

Gerontology 2017;63:495-506

DOI: $10.1159 / 000478263$
Hauer/Ullrich/Dutzi/Beurskens/Kern/ Bauer/Schwenk 
lowing proper progression of intensity, and the introduction of safety rules. Particularly, the weekly phone contact may have played a major role in adherence as it represented an ongoing and reliable personal contact with multiple options to interact and to strengthen adherence and personal commitment.

Results indicate that a high training adherence can be achieved despite relevant cognitive and advanced functional impairment. No training-induced adverse events occurred (i.e., no falls or injuries during exercises), indicating that even challenging exercises, as included in the presented program (e.g., balance tasks, STS training, stair rise), did not lead to an increased risk in patients with a high risk for falls. Thus, we consider the home-based training program safe and feasible to be implemented at patients' homes following ward-based rehabilitation.

\section{Effects of Home-Based Training on Motor}

\section{Performance}

Our home-based intervention significantly increased key motor performances as indicated by results of the SPPB and decreased deficits in gait and balance as indicated by results of POMA. Training effects seem specific as performances specifically improved in exercises trained in our home-based program (i.e., balance, STS) compared to those on which the training did not specifically focus on (i.e., walking). These findings comply with the well-documented principle of training specificity [37]. The documented training effects are relevant for ADL competence as they were achieved in key motor performances such as postural control or transfer tasks, representing major competences in everyday life. In addition, deficits in such key motor performances (especially STS transfers) represent high-impact risk factors for falls in a multimorbid population with CI with a particular risk for falls [38].

The program led to high training effects as indicated by large effect sizes in this comparatively small study sample. Findings are in line with studies on supervised groupbased physical exercise in cognitively impaired persons $[15,39]$. The high impact of our home-based training using simple and low-cost interventions provides evidence that the potential of patients included in ward-based rehabilitation may currently not be fully exploited. In addition, study results confirm that training in persons with low basic performance levels is highly rewarding when an individually tailored program with adequate progression of training intensity and difficulty is applied $[8,20]$.

Training effects were assessed by different assessment strategies but almost all indicated improvements in func-

Home Training in Cognitively Impaired Patients tional performance. We used well-established clinical assessment methods (POMA, SPPB) [29, 30], developed for persons with motor impairment and validated in subsamples of persons with CI, as well as advanced methods based on body-fixed sensors [33]. The latter allow reliable insight into relevant functional performances not ascertainable by established clinical measures. In our study, body-fixed sensors were used to evaluate their potential to document training effects on STS performances with high sensitivity to change as a major issue in intervention studies. Using technically driven measures, we were able to detect significant effects in a number of parameters, although we only used a small sample with advanced functional deficits. The extraordinary large effect sizes found for the sensor-based assessments during STS $\left(\right.$ eta $\left.^{2}=0.35-0.55\right)$ highlight their potential to sensitively document interventional effects, providing detailed insight into biomechanics of the STS transfer (i.e., flexion/ extension ranges, angular velocities), a motor performance measure with high relevance for future specification and optimization of training interventions. As shown in Table 4, duration of a major phase of the transfer task (movement of trunk to create a sufficient momentum for upper-forward motions) as well as related ankle velocities were positively affected by the training intervention. Further, such sensor-based biomechanical measures have been shown to be of greater clinical relevance compared to the manually recorded STS measures in older adults (i.e., instrumented STS times were associated with participants' health status, while manually recorded STS times were not) [40].

\section{Effects of Home-Based Training on Physical Activity}

Previous research on home-based training in patients with CI lacks objective measures of physical performance and focused on functional decline instead [8]. However, it is crucial to increase the sustainability of performance benefits when formal rehabilitation programs end. The key to such efforts is behavioral modifications to increase physical activity, leading to higher sustainability of training effects as achieved during ward-based rehabilitation. In our study, we documented physical activity to evaluate the effect of the intervention in a highly sedentary study sample, severely affected by motor and cognitive impairments often associated with specific symptoms (i.e., apathy, spatiotemporal disorientation or risk of falling limiting outdoor activities, or decreased adherence to interventional programs) [38]. In contrast to previous studies $[8,13]$, we were able to show that physical activity can be increased in the vulnerable population of CI patients us-

Gerontology 2017;63:495-506

DOI: $10.1159 / 000478263$ 
ing home-based exercise programs, as documented by a comprehensive questionnaire developed and successfully validated in patients with CI [31].

\section{Effects of Immediate versus Delayed Home-Based}

\section{Training}

The design of this pilot study allowed the investigation of the effect of a delayed onset of the post-ward training, a factor which, to the best of our knowledge, has not been investigated with regard to post-ward rehabilitation programs in patients with CI yet. The transition from the hospital to the home environment is often delayed. Reasons are manifold and range from reconstruction works at the patients' homes to install disability-friendly environments to short-term care in nursing homes. A recent systematic review on post-ward adherence to rehabilitation programs further indicated that an early onset of care (i.e., early medical consultations and appointments with medical staff as well as timely self-monitoring of activity following hospital discharge) improved uptake of and adherence to rehabilitation programs in cardiac patients [41] and yielded more pronounced training benefits compared to a delayed onset of training [23,24]. In line with these findings, results of our study showed that adherence rates significantly decreased when post-ward training is delayed by 6 weeks. Patients in the delayed training group only conducted half of the sessions compared to the group with immediate onset. This deficit in participation was also mirrored in the functional performances of patients. Although the sample size in our study was rather small and both groups increased performance following training, effect sizes indicate that the majority of functional outcomes further improved following an immediate onset of training. That is, performance improvements in SPPB and POMA were more pronounced following an immediate start of the training. Thus, postward rehabilitation programs should be implemented as soon as possible following hospital discharge to fully exploit the rehabilitation potentials of patients with CI.

\section{Limitations}

The major limitation of the current study is its small sample size, reducing the statistical power to identify significant effects. However, significant improvements and trends as achieved in this study, associated with the large effect sizes found, indicate a high effectiveness of the training program in this vulnerable, multimorbid, and cognitively impaired patient group. Further, based on the study design (wait list control design), no follow-up data were available for the training groups. Thus, retention of training results could not be obtained from our data set.

\section{Conclusions}

Results of this pilot study document high effectiveness of a post-ward, home-based training program specifically tailored for patients with CI. Thus, the implementation of home-based interventions involving training of key motor performances seems feasible and valuable in this vulnerable patient group, particularly when implemented shortly after hospital discharge. Further, innovative, sensor-based assessment tools are suitable to extend established clinical measurements, allow new insights into the biomechanics of key motor performances and may be used to further specify and optimize training interventions in cognitively impaired patients.

\section{Acknowledgements}

We would like to thank the Ministry of Social Affairs of BadenWürttemberg e.V. for financial support of the study and Michaela Günther for her support in data assessment.

\section{Statement of Ethics}

The study conforms to the latest version of the Helsinki Declaration and was approved by the Ethics Committee of the Medical Department at the University of Heidelberg.

\section{Disclosure Statement}

The authors declare that there is no conflict of interest associated with this work.

\section{Funding Sources}

The study was funded by a research grant from the Ministerium für Arbeit und Sozialordnung, Familie, Frauen und Senioren, Baden-Württemberg (Ministry of Social Affairs of the State of Baden-Württemberg, Germany) and support by the "gesetzlichen Pflegeversicherung” (nursing care insurance, Germany) following $\$ 45$ c, Abs.45 SGB XI to K.H.

\section{Author Contributions}

K.H.: study concept, design, and management, supervision of data collection, statistical design and analysis, interpretation of data, and preparation of manuscript. P.U.: acquisition of participants, study management, data analysis, interpretation of data, and preparation of manuscript. R.B.: preparation of manuscript, data analysis. All authors contributed to interpretation of data, drafting the article, and final approval of the version to be published. 


\section{References}

1 von Renteln-Kruse W, Neumann L, Klugmann B, Liebetrau A, Golgert S, Dapp U, Frilling B: Geriatric patients with cognitive impairment. Dtsch Arztebl Int 2015;112:103112.

2 Njegovan V, Hing MM, Mitchell SL, Molnar FJ: The hierarchy of functional loss associated with cognitive decline in older persons. J Gerontol A Biol Sci Med Sci 2001;56:M638M643.

3 Luppa M, Riedel-Heller SG, Luck T, Wiese B, van den Bussche $H$, Haller F, Sauder M, Mosch E, Pentzek M, Wollny A, et al: Agerelated predictors of institutionalization: results of the German study on ageing, cognition and dementia in primary care patients (AgeCoDe). Soc Psychiatry Psychiatr Epidemiol 2012;47:263-270.

4 Seematter-Bagnoud L, Lecureux E, Rochat S, Monod S, Lenoble-Hoskovec C, Bula CJ: Predictors of functional recovery in patients admitted to geriatric postacute rehabilitation. Arch Phys Med Rehabil 2013;94:2373-2380.

5 Chenoweth L, Kable A, Pond D: Research in hospital discharge procedures addresses gaps in care continuity in the community, but leaves gaping holes for people with dementia: a review of the literature. Australas J Ageing 2015;34:9-14.

6 Poynter L, Kwan J, Sayer A, Vassallo M: Do cognitively impaired patients benefit from rehabilitation? Rev Clin Gerontol 2008;18:5964.

7 Rantakokko M, Iwarsson S, Manty M, Leinonen R, Rantanen T: Perceived barriers in the outdoor environment and development of walking difficulties in older people. Age Ageing 2012;41:118-121.

8 Suttanon P, Hill KD, Said CM, Williams SB, Byrne KN, LoGiudice D, Lautenschlager NT, Dodd KJ: Feasibility, safety and preliminary evidence of the effectiveness of a home-based exercise programme for older people with Alzheimer's disease: a pilot randomized controlled trial. Clin Rehabil 2013;27:427-438.

9 Pitkala KH, Poysti MM, Laakkonen ML, Tilvis RS, Savikko N, Kautiainen H, Strandberg TE: Effects of the Finnish Alzheimer disease exercise trial (FINALEX): a randomized controlled trial. JAMA Intern Med 2013;173: 894-901.

10 Teri L, Gibbons LE, McCurry SM, Logsdon RG, Buchner DM, Barlow WE, Kukull WA, LaCroix AZ, McCormick W, Larson EB: Exercise plus behavioral management in patients with Alzheimer disease: a randomized controlled trial. JAMA 2003;290:2015-2022.

11 Prick AE, de Lange J, Scherder E, Twisk J, Pot AM: The effects of a multicomponent dyadic intervention on the mood, behavior, and physical health of people with dementia: a randomized controlled trial. Clin Interv Aging 2016;11:383-395.
12 Steinberg M, Leoutsakos JM, Podewils LJ, Lyketsos CG: Evaluation of a home-based exercise program in the treatment of Alzheimer's disease: the Maximizing Independence in Dementia (MIND) study. Int J Geriatr Psychiatry 2009;24:680-685.

13 Wesson J, Clemson L, Brodaty H, Lord S, Taylor M, Gitlin L, Close J: A feasibility study and pilot randomised trial of a tailored prevention program to reduce falls in older people with mild dementia. BMC Geriatr 2013;13:89.

14 Schwenk M, Dutzi I, Englert S, Micol W, Najafi B, Mohler J, Hauer K: An intensive exercise program improves motor performances in patients with dementia: translational model of geriatric rehabilitation. J Alzheimers Dis 2014;39:487-498.

15 Hauer K, Schwenk M, Zieschang T, Essig M, Becker C, Oster P: Physical training improves motor performance in people with dementia: a randomized controlled trial. J Am Geriatr Soc 2012;60:8-15.

16 Fairhall N, Sherrington C, Kurrle SE, Lord SR Lockwood K, Cameron ID: Effect of a multifactorial interdisciplinary intervention on mobility-related disability in frail older people: randomised controlled trial. BMC Med 2012;10:120.

17 Karlsson A, Berggren M, Gustafson Y, Olofsson B, Lindelof N, Stenvall M: Effects of geriatric interdisciplinary home rehabilitation on walking ability and length of hospital stay after hip fracture: a randomized controlled trial. J Am Med Dir Assoc 2016;17:464 e469-e464 e415.

18 Moseley AM, Sherrington C, Lord SR, Barraclough E, St George RJ, Cameron ID: Mobility training after hip fracture: a randomised controlled trial. Age Ageing 2009;38:74-80.

19 Shyu YI, Tsai WC, Chen MC, Liang J, Cheng HS, Wu CC, Su JY, Chou SW: Two-year effects of an interdisciplinary intervention on recovery following hip fracture in older Taiwanese with cognitive impairment. Int J Geriatr Psychiatry 2012;27:529-538.

20 Sherrington C, Lord SR, Vogler CM, Close JC, Howard K, Dean CM, Heller GZ, Clemson L, O’Rourke SD, Ramsay E, et al: A post-hospital home exercise program improved mobility but increased falls in older people: a randomised controlled trial. PLoS One 2014;9: e104412.

21 Jackson C, Shahsahebi M, Wedlake T, DuBard CA: Timeliness of outpatient follow-up: an evidence-based approach for planning after hospital discharge. Ann Fam Med 2015;13: 115-122.

22 Macchi C, Fattirolli F, Lova RM, Conti AA Luisi ML, Intini R, Zipoli R, Burgisser C, Guarducci L, Masotti G, et al: Early and late rehabilitation and physical training in elderly patients after cardiac surgery. Am J Phys Med Rehabil 2007;86:826-834.
23 Fell J, Dale V, Doherty P: Does the timing of cardiac rehabilitation impact fitness outcomes? An observational analysis. Open Heart 2015;3:e000369.

24 Haykowsky M, Scott J, Esch B, Schopflocher D, Myers J, Paterson I, Warburton D, Jones L, Clark AM: A meta-analysis of the effects of exercise training on left ventricular remodeling following myocardial infarction: start early and go longer for greatest exercise benefits on remodeling. Trials 2011;12:92.

25 Kitwood T: The dialectics of dementia: with particular reference to Alzheimer's disease. Ageing Soc 1990;10:177-196.

26 Huger D, Zieschang T, Schwenk M, Oster P, Becker C, Hauer K: Designing studies on the effectiveness of physical training in patients with cognitive impairment. Z Gerontol Geriatr 2009;42:11-19.

27 Yesavage JA, Brink TL, Rose TL, Lum O, Huang V, Adey M, Leirer VO: Development and validation of a geriatric depression screening scale: a preliminary report. J Psychiatr Res 1982;17:37-49.

28 Folstein MF, Folstein SE, McHugh PR: "Minimental state.” A practical method for grading the cognitive state of patients for the clinician. J Psychiatr Res 1975;12:189-198.

29 Guralnik JM, Simonsick EM, Ferrucci L, Glynn RJ, Berkman LF, Blazer DG, Scherr PA, Wallace RB: A short physical performance battery assessing lower extremity function: association with self-reported disability and prediction of mortality and nursing home admission. J Gerontol 1994;49:M85-M94.

30 Tinetti ME: Performance-oriented assessment of mobility problems in elderly patients. J Am Geriatr Soc 1986;34:119-126.

31 Hauer K, Lord SR, Lindemann U, Lamb SE, Aminian K, Schwenk M: Assessment of physical activity in older people with and without cognitive impairment. J Aging Phys Act 2011; 19:347-372.

32 Zieschang T, Schwenk M, Becker C, Oster P, Hauer K: Feasibility and accuracy of fall reports in persons with dementia: a prospective observational study. Int Psychogeriatr 2012; 24:587-598.

33 Schwenk M, Gogulla S, Englert S, Czempik A, Hauer K: Test-retest reliability and minimal detectable change of repeated sit-to-stand analysis using one body fixed sensor in geriatric patients. Physiol Meas 2012;33:19311946.

34 Cohen J: Statistical Power for the Behavioral Sciences. Hillsdale, Erlbaum, 1988.

35 Moher D, Hopewell S, Schulz KF, Montori V, Gotzsche PC, Devereaux PJ, Elbourne D, Egger M, Altman DG; CONSORT: CONSORT 2010 explanation and elaboration: updated guidelines for reporting parallel group randomised trials. Int J Surg 2012;10:28-55. 
36 Kwon S, Perera S, Pahor M, Katula JA, King AC, Groessl EJ, Studenski SA: What is a meaningful change in physical performance? Findings from a clinical trial in older adults (the LIFE-P study). J Nutr Health Aging 2009; 13:538-544.

37 Hawley JA: Adaptations of skeletal muscle to prolonged, intense endurance training. Clin Exp Pharmacol Physiol 2002;29:218-222.
38 Harlein J, Dassen T, Halfens RJ, Heinze C: Fall risk factors in older people with dementia or cognitive impairment: a systematic review. J Adv Nurs 2009;65:922-933.

39 Heyn PC, Johnson KE, Kramer AF: Endurance and strength training outcomes on cognitively impaired and cognitively intact older adults: a meta-analysis. J Nutr Health Aging 2008;12:401-409.
40 van Lummel RC, Walgaard S, Maier AB, Ainsworth E, Beek PJ, van Dieen JH: The Instrumented Sit-to-Stand Test (iSTS) has greater clinical relevance than the manually recorded Sit-to-Stand Test in older adults. PLoS One 2016;11:e0157968.

41 Karmali KN, Davies P, Taylor F, Beswick A, Martin N, Ebrahim S: Promoting patient uptake and adherence in cardiac rehabilitation. Cochrane Database Syst Rev 2014;CD007131. 